\title{
INIBIDORES DE ENZIMAS DIGESTIVAS DE INSETOS-PRAGA
}

\author{
Inhibitors of Digestive Enzymes of Insect Pests
}

\author{
Alberto Luiz Marsaro Júnior ${ }^{1}$ \\ Sonia Maria Noemberg Lazzari ${ }^{2}$ \\ Airton Rodrigues Pinto Júnior ${ }^{3}$
}

\section{Resumo}

Os insetos fitófagos, como muitos outros animais, utilizam-se de enzimas digestivas, tais como amilases e proteinases para processar os nutrientes obtidos das plantas, necessários para seu desenvolvimento. As plantas, por sua vez, utilizam diversos mecanismos para se defender do ataque dos insetos, dentre eles a produção de proteínas que inibem a ação das enzimas digestivas dos insetos. Os inibidores de enzimas digestivas podem ser encontrados em diversas partes das plantas, mas os órgãos de reserva, sementes e tubérculos são as principais fontes dessas proteínas. Estudos recentes têm demonstrado que a presença dos inibidores de enzimas digestivas nas plantas aumenta sua resistência ao ataque das pragas. Nem sempre os inibidores estão em plantas de interesse comercial ou quando estão não apresentam níveis suficientes para evitar os danos das pragas. A biotecnologia tem contribuído para a solução desse problema por meio da inserção dos genes responsáveis pela expressão dos inibidores das enzimas digestivas nas plantas de interesse econômico. Os estudos experimentais demonstram que as plantas geneticamente modificadas expressando essas proteínas têm se mostrado mais resistentes às pragas que as plantas não transgênicas. A adoção dessa tecnologia poderá reduzir o uso de inseticidas, minimizando os riscos para a saúde humana e animal. Antes, porém, da liberação dessas plantas em escala comercial, diversas pesquisas necessitam ser realizadas com o objetivo de avaliar os possíveis impactos que essa nova tecnologia pode causar ao meio ambiente e à saúde do homem e dos animais.

Palavras-chave: Amilases; Pragas agrícolas; Proteinases; Resistência de plantas.

1 Entomologista, Embrapa Roraima, Br174, km 8, Distrito Industrial, CP 133, CEP 69301-970, Boa Vista/RR, alberto@cpafrrembrapa.br. Depto. de Zoologia, UFPR, CP 19020, CEP 81531-980, Curitiba/PR, lazzari@ufpr.br.

3 Centro de Ciências Agrárias, PUCPR, BR 376, km 14, CP 129, CEP 83010-500, São José dos Pinhais/PR, airton.junior@pucpr.br. *Autor para correspondência. 


\section{Abstract}

Phytophagous insects and other animals use digestive enzymes, such as amylases and proteinases to process the nutrients obtained from the plants necessary for their development. The plants, on the other hand, developed several mechanisms to defend themselves from the attack of insect pests, including the production of proteins that inhibit the action of digestive enzymes of insects. The digestive enzyme inhibitors can be found in several parts of the plants, but the reserve organs, such as seeds and tubers, are the main sources of those proteins. Recent studies have demonstrated that the presence of these compounds in the plants increases their resistance to the attack of pests. The inhibitors are not always present in plants of commercial value or may not be present in levels high enough to avoid the damages of the pests. The biotechnology has contributed to the solution of this problem by inserting the genes responsible for the expression of the digestive enzyme inhibitors in the plants of economical interest. The experimental studies demonstrate that the genetically modified plants expressing those proteins are more resistant to the pests than the non transgenic plants. The adoption of this technology can reduce the use of insecticides and minimize the risks for the human and animal health. However, before the liberation of these plants in commercial scale, various research need to be done with the objective of evaluating the possible impact that this new technology can possibly influence to the environment and to the human and health.

Keywords: Amylases; Insect pests; Proteinases; Plant resistance.

\section{Enzimas Digestivas}

Como a maioria dos organismos vivos, os insetos também produzem enzimas digestivas para a obtenção dos nutrientes essenciais às suas atividades metabólicas. Das 14 enzimas digestivas utilizadas pelos insetos, 12 já foram caracterizadas em diferentes espécies por pesquisadores da ESALQ-USP (MEDEIROS, 2000). Dentre essas, as mais importantes e que têm sido mais investigadas são as a-amilases e proteinases.

As $\alpha$-amilases constituem uma família de endoamilases que catalisam a hidrólise de ligações glicosídicas $\alpha-1,4$ do amido, glicogênio e outros carboidratos (FRANCO et al., 2002). Essas enzimas são muito importantes para insetos e fungos, especialmente para aqueles que se desenvolvem em grãos ricos em amido.

As proteinases são enzimas extracelulares presentes no lúmem do intestino dos insetos, atuando no metabolismo de aminoácidos essenciais. Essas enzimas são classificadas de acordo com a União Internacional de Bioquímica em quatro grandes classes: proteinases serínicas, cisteínicas, aspárticas e metalo-proteinases. As mais estudadas são as proteinases serínicas, muito freqüentes em vários insetos e que se assemelham à tripsina $\mathrm{e}$ à quimiotripsina de mamíferos. As proteinases cisteínicas também são importantes enzimas digestivas para os insetos Callosobru chus maculatus (Fabricius) e Acanthoscelides obtectus (Say) (Coleoptera: Bruchidae) (FRANCO et al., 1999).

\section{Fontes dos Inibidores de Enzimas Digestivas}

As plantas, no decorrer da evolução, desenvolveram mecanismos de resistência, produzindo nos tecidos atacados (folhas, frutos e órgãos de reserva) inibidores para as enzimas digestivas dos insetos fitófagos.

Os principais inibidores de a-amilase conhecidos são os encontrados no feijão (Phaseolus vulgaris), trigo (Triticum aestivum) e milho (Zea mays). Os inibidores do feijão são identificados como a-AI1 e a-AI2 e os do trigo foram denominados de 0,19 e 0,53 (Franco et al., 1999). No milho já foram identificadas quatro proteínas inibidoras de amilase com os seguintes pesos moleculares: $12 \mathrm{kDa}$ (Blanco-Labra et al., 1995 ), 19,7 kDa (Figueira et al., 2003a), 23,8 kDa (Figueira et al., 2003b) e 29,6 kDa (Blanco-Labra; IturbeChiñas, 1981).

Os principais inibidores de proteinase conhecidos e suas respectivas fontes são: CII (inibidor de proteinase serínica presente na soja Glycine max), CpTI (inibidor de tripsina do feijãode-corda Vigna unguiculata), OC I (inibidor de cisteína do arroz Oryza sativa), NaPI (inibidor de protease de Nicotiana alata), Pot PI I (inibidor de proteinase I da batata Solanum tuberosum) e Pot PI II (inibidor de proteinase II da batata S. tuberosum) (FRANCO et al., 1999). 


\section{Atividade dos Inibidores de Enzimas Digestivas}

Os mecanismos de interação e especificidade dos inibidores com as enzimas digestivas são complexos e ainda não foram completamente elucidados. Nos inibidores do tipo não proteináceos, a atividade inibitória contra as $\alpha$-amilases é devida em parte à sua estrutura cíclica que se assemelha aos substratos da a-amilase e, por isso, possibilita a ligação nos sítios catalíticos desta enzima (FRANCO et al., 2002). Uma vez que a enzima é inibida, a assimilação de nutrientes pelos insetos é reduzida e, conseqüentemente, o desenvolvimento dessas pragas é afetado.

$O$ inibidor do feijão, $\alpha$-AI1, inibe as $\alpha$ amilases de importantes insetos-praga, como: Tribolium castaneum (Herbst), Tribolium confusum Duval e Tenebrio molitor Linnaeus (Coleoptera: Tenebrionidae) e Sitophilus oryzae (Linnaeus) (Coleoptera: Curculionidae) (Pueyo et. al., 1995); C. maculatus, Callosobruchus chinensis e Callosobruchus analis (Fabricius) (Coleoptera: Bruchidae) (Ishimoto et al., 1996); Diabrotica vergifera vergifera LeConte (Coleoptera: Chrysomelidae) (Titarenko; Chrispeels, 2000) e Hypothenemus hampei (Ferrari) (Coleoptera: Scolytidae) (Valencia et al., 2000). Enquanto o $\alpha$-AI2, presente nessa mesma leguminosa, inibe as $\alpha$-amilases de Zabrotes subfasciatus Boheman (Coleoptera: Bruchidae) (GROSSI De SÁ; CHRISPEELS, 1997).

O inibidor do trigo, 0,53, por sua vez, inibe as $\alpha$-amilases de A. obtectus, Z. subfasciatus e C. maculatus (Franco et al., 1999).

O primeiro estudo com os inibidores de $\alpha$-amilase em milho foi realizado por Blanco-Labra e Iturbe-Chiñas (1981). Os autores verificaram que a proteína de 29,6 kDa inibia as $\alpha$-amilases de Sitophilus zeamais Motschulsky (Coleoptera: Curculionidae), Rhyzopertha dominica (Fabricius) (Coleoptera: Bostrichidae) e T. castaneum.

Posteriormente, também no milho, Blanco-Labra et al. (1995) verificaram que a proteína inibidora de $12 \mathrm{kDa}$ inibia as $\alpha$-amilases de T. castaneum e C. maculatus e as proteinases de Prostephanustruncatus (Horn) (Coleoptera: Bostrichidae).

Recentemente, foram descobertos dois novos inibidores de $\alpha$-amilase em milho. $O$ de 19,7 $\mathrm{kDa}$ inibe as $\alpha$-amilases de S. zeamais, P. truncatus, A. obtectus e Z. subfasciatus (FIGUEIRA et al., 2003a). O outro, de 23,8 kDa, inibe as $\alpha$-amilases de A. obtectus, Z. subfasciatus, T. castaneum e Sitotroga cerealella (Olivier) (Lepidoptera: Gelechiidae) (FIGUEIRA et al., 2003b).

Além disso, proteínas presentes no milho também inibiram o crescimento de importantes fungos fitopatogênicos e micotoxigênicos presentes em grãos, Aspergillus flavus, Aspergillus parasiticus, Fusarium graminearum, Fusarium moniliforme, Cercospora kikuchii, Penicillium chrysogenum e Fusarium verticillioides (CHEN et al., 1999a; CHEN et al., 1999b; FIGUEIRA et al., 2003a; FIGUEIRA et al., 2003b).

Estudos recentes demonstraram que os inibidores de amilase, presentes em grãos de diferentes híbridos de milho, correlacionaram-se significativamente com o ciclo biológico de $\mathrm{S}$. zeamais $(0,61$ com $\mathrm{p}<0,05)$ e com um importante parâmetro que mede a suscetibilidade dos grãos ao ataque desse inseto, 0 índice de suscetibilidade, $(-0,58$ com $p<0,05)$ (MARSARO JÚNIOR, 2004; MARSARO JÚNIOR et al., 2004). Esses resultados indicam que a presença dos inibidores de amilase contribui para aumentar a resistência dos híbridos de milho contra o ataque de S. zeamais.

Alguns problemas inviabilizavam o uso dos inibidores de enzimas digestivas no Manejo Integrado de Pragas (MIP), destacando-se: 1) As plantas de interesse comercial, na maioria das vezes, não apresentavam os inibidores das enzimas digestivas das pragas; 2) Quando apresentavam não estavam em níveis suficientes para inativar as enzimas e; 3) Os inibidores de enzimas digestivas geralmente se encontravam em plantas que não eram de interesse comercial, como, por exemplo, feijão-de-corda (V. unguiculata).

Os pesquisadores têm resolvido esses problemas pela engenharia genética, utilizando técnicas de biologia molecular para identificar e isolar os genes responsáveis pela expressão dos inibidores de enzimas digestivas de insetos ou fungos, transferindo-os em seguida para o genoma da planta de interesse comercial, resultando assim nas plantas transgênicas.

\section{Plantas Transgênicas Expressando Inibidores de Enzimas Digestivas}

A utilização de plantas transgênicas expressando inibidores de enzimas digestivas tem sido 
considerada como uma medida eficiente no controle de pragas. Ervilhas transgênicas expressando o inibidor de $\alpha$-amilase $\alpha$-AI1 foram altamente resistentes às pragas $\mathrm{C}$. maculatus $\mathrm{e}$ C. chinensis (Shade et al., 1994) e à praga Bruchus pisorum (Coleoptera: Bruchidae) (Schroeder et al., 1995). Utilizando esse mesmo inibidor, Ishimoto et al. (1996) obtiveram feijões azuki resistentes a $\mathrm{C}$. chinensis.

Duan et al. (1996) demonstraram que arroz transgênico, expressando o inibidor de proteinase II da batata, é mais resistente à praga Sesamia inferens (Lepidoptera: Noctuidae) que 0 arroz não transgênico. Cana-de-açúcar transgênica, contendo os inibidores de proteinase da soja (Kunitz; Bowman-Birk), tem sido resistente à praga Diatraea saccharalis (Lepidoptera: Pyralidae), em estudos desenvolvidos na ESALQ - USP (MEDEIROS, 2000).

Os insetos, contudo, podem adaptarse aos inibidores de enzimas digestivas e não serem mais afetados por eles. Dois fatores podem contribuir para essa adaptação: a mutação que resulta em enzimas insensíveis aos inibidores e a síntese de enzimas capazes de inativar ou degradar os inibidores (SILVA-FILHO; FALCO, 2000).

A proposta dos pesquisadores para contornar essa adaptação é fazer com que as plantas expressem mais de um tipo de inibidor de enzimas digestivas e/ou gerarem novas moléculas de inibidores que sejam mais potentes e específicas contra as enzimas digestivas dos insetos.

\section{Conclusões}

O uso de plantas, geneticamente modificadas ou não, expressando inibidores de enzimas digestivas de insetos, poderá reduzir 0 uso de inseticidas, minimizando, assim, os riscos para a saúde humana e animal.

Antes, porém, da liberação dessas plantas em escala comercial, diversas pesquisas necessitam ser realizadas com o objetivo de avaliar os possíveis impactos que essa nova tecnologia pode causar ao meio ambiente e à saúde do homem e dos animais.

\section{Referências}

BLANCO-LABRA, A. et al. Further characterization of the $12 \mathrm{kDa}$ protease/alphaamylase inhibitor present in maize seeds. Journal of Food Biochemistry, Trumbull, v.19, p.27-41, 1995.

BLANCO-LABRA, A.; ITURBE-CHIÑAS, F. A. Purification and characterization of an a-amylase inhibitor from maize (Zea maize). Journal of Food Biochemistry, Trumbull, v.5, p.1-17, 1981.

CHEN, Z.Y. et al. Inhibition of plant-pathogenic fungi by a corn trypsin inhibitor overexpressed in Escherichia coli. Applied and Environmental Microbiology, Washington, v. 65, n. 3, p. 13201324, 1999a.

. etal. A com trypsin inhibitor with antifungal activity inhibits Aspergillus flavus a-amylase. Phytopathology, St. Paul, v. 89, n. 10, 1999b.

DUAN, X. etal. Transgenic rice plants harboring an introduced potato proteinase inhibitor gene II are insectresistant. Nature Biotechnology, New York, v.14, p.494-498, 1996.

FIGUEIRA, E. L Z. et al. Amylase inhibitor present in corn seeds active in vitro against amylase from Fusarium verticillioides. Plant Disease, St. Paul, v.87, n.3, p.233-240, 2003b.

FIGUEIRA, E. L. Z. et al. Characterization of an hydrophobic amylase inhibitor from corn (Zea mays) seeds with activity against amylase from Fusarium verticillioides. Phytopathology, St. Paul, v.93, n.8, p. 917-922, 2003a.

FRANCO, O. L et al. Resistência de plantas a insetos. Biotecnologia Ciência \& Desenvolvimento, Brasília, n. 11, p. 36-40, 1999.

Plant a-amylase inhibitors and their interaction with insect $\alpha$-amylases. European Journal of Biochemistry, Paris, v. 269, p. 397412, 2002.

GROSSI de SA, M. F.; CHRISPEELS, M. J. Molecular cloning of bruchid (Zabrotessubfaciatus) $\alpha$-amylase cDNA and interactions of the expressed enzyme with bean amylase inhibitors. Insect Biochemistry and Molecular Biology, Oxon, v. 27, n. 4, p. 271281, 1997. 
ISHIMOTO, M. etal. Bruchid resistance of transgenic azuki bean expressing seed alpha-amylase inhibitor of common bean. Entomologia Ex perimentallis et Applicata, Dordrecht, v. 79, n. 3, p. 309-315, 1996.

MARSARO JÚNIOR, A.L Resistência de genótipos de milho, Zea mays L. (Poaceae), ao ataque de Sitophilus zeamais Motschulsky (Coleoptera: Curculionidae). 2004. 84 f. Tese (Doutorado em Entomologia) - Setor de Ciências Biológicas, Universidade Federal do Paraná. Curitiba, 2004.

MARSARO JÚNIOR, A. L et al. Inibidores de amilase em híbridos de milho como fator de resistência ao ataque de Sitophilus zeamais Motschulsky (Coleoptera: Curculionidae). In: CONGRESSO BRASILEIRO DE ENTOMOLOGIA, 20, 2004, Gramado. Resumos... Gramado: SEB, 2004. p.586.

MEDEIROS, M. A. As pragas também morrem pela boca. Pesquisa FAPESP, São Paulo, SP: p.20-27, jun. 2000.

PUEYO, J. J. et al. Effects of bean and wheat aamylase inhibitors on a-amylase activity and growth of stored product insect pests. Entomologia Experimentallis et Applicata, Dordrecht, v. 74, p. 237-244, 1995.
SCHROEDER, H. E. et al. Bean a-amylase inhibitor confers resistance to the pea weevil (Bruchus pisorum) in transgenic peas (Pisum sativum L.). Plant Physiology, Rockville, v.107, p.1233-1239, 1995.

SHADE, R. E. etal. Transgenic pea seeds expressing the alpha-amylase inhibitor of the common bean are resistant to bruchid beetles. Bio Technology, New York, v. 12, n. 8, p.793-796, 1994.

SILVA-FILHO, M. C.; FALCO, M. C. Interação planta-inseto. Biotecnologia Ciência \& Desenvolvimento, Brasília, n.12, p.38-42, 2000.

TITARENKO, E.; CHRISPEELS, M. J. cDNA cloning, biochemical characterization and inhibition by plant inhibitors of the a-amylases of the Western corn rootworm, Diabrotica virgifera virgifera. Insect Biochemistry and Molecular Biology, Oxon, v.30, p.979-990, 2000.

VALENCIA, A.; BUSTILLO, A. E.; OSSA, G. E.; CHRISPEELS, M. J. a-Amylases of the coffee berry borer (Hypothen emus hampei) and their inhibition by two plant amylase inhibitors. Insect Biochemistry and Molecular Biology, Oxon, v.30, p.207-213, 2000. 